\title{
CORRECTIONS
}

\section{Online marketing of medical procedures needs better regulation}

This Personal View mistakenly cited only Richard Smith as the author (BMJ 2012;344:e1399, doi:10.1136/bmj.e1399). The full list of authors should include Neil Lunt, Department of Social Policy and Social Work, University of York, York, UK and
Johanna Hanefeld, School of Social and Political Science; University of Edinburgh, Edinburgh, UK.

Cite this as: BMJ 2012;344:e1711

๑ BMJ Publishing Group Ltd 2012 